JOURNAL CLUB

\title{
ERK3 in lung cancer cell invasion
}

ERK3, an atypical mitogen-activated protein kinase, was first identified 20 years ago, and its role in cancer development is yet to be fully elucidated. Steroid receptor coactivator 3 (SRC-3) has been well described in a number of different cancers, including lung cancer, as a nuclear receptor coactivator of PEA3-regulated MMP expression to promote cancer cell invasion.

The authors use immunoprecipitation analysis in H1299 lung cancer cells to identify the oncogene SRC-3 as a substrate of the ERK3-signalling cascade for phosphorylation at serine 857(S857). Additionally, overexpression of ERK3 and SRC-3 was shown to enhance MMP2 activity, while mutation of S857, as well as knockdown of ERK3 and SRC-3, inhibited MMP2 expression. Phosphorylation of S857 was an essential step in encouraging interaction of SRC-3 and PEA3 and occupancy at the MMP2 promoter.

A 7.4-fold increased expression of ERK3 mRNA was noted in squamous cell lung carcinoma compared with normal lung tissue, which was confirmed with immunohistochemistry. Further experiments using mice models with ERK3/SRC-3 knockdown indicated eliminated invasion potential of $\mathrm{H} 1299$ cells, with no lung cancer cell colonisation seen after 5 weeks.

This study works towards increasing understanding of the ERK3 signalling pathway: phosphorylation of SRC-3 at S857 and MMP expression via PEA coactivation. The clinical implications of upregulation of ERK3 in squamous cell lung cancer, and its role in promoting cell invasion, may provide a new potential therapeutic target for this cancer sub-group.

- Long W, Foulds CE, Qin J, et al. ERK3 signals through SRC-3 coactivator to promote human lung cancer cell invasion. J Clin Invest 2012;122:1869-80.

\section{Arthee Yogendran}

Correspondence to Dr Arthee Yogendran, FY2, Medway NHS Foundation Trust, Department of Thoracic Medicine, Windmill Road, Gillingham ME7 5NY, UK; artheey@doctors.org.uk

Provenance and peer review Not commissioned; internally peer reviewed.

To cite Yogendran A. Thorax 2013;68:379.

Published Online First 16 July 2012

Thorax 2013;68:379. doi:10.1136/thoraxjnl-2012-202322 